\title{
Differentiation of regioisomeric chloroamphetamine analogs using gas chromatography-chemical ionization-tandem mass spectrometry
}

\author{
Shoko Negishi ${ }^{1,2} \cdot$ Yukiko Nakazono $^{1,2}$ - Yuko T. Iwata ${ }^{1}$ 'Tatsuyuki Kanamori ${ }^{1}$. \\ Kenji Tsujikawa $^{1} \cdot$ Kenji Kuwayama $^{1} \cdot$ Tadashi Yamamuro $^{1} \cdot$ Kazuna Miyamoto $^{2,3}$. \\ Takuya Yamashita ${ }^{3} \cdot$ Fumiyo Kasuya $^{3} \cdot$ Hiroyuki Inoue $^{1}$
}

Received: 4 February 2015/ Accepted: 20 April 2015/Published online: 19 May 2015

(C) Japanese Association of Forensic Toxicology and Springer Japan 2015

\begin{abstract}
In recent years, a large number of clandestinely synthesized new psychoactive substances with high structural variety have been detected in forensic samples. Analytical differentiation of regioisomers is a significant issue in forensic drug analysis, because, in most cases, legal controls are placed on only one or two of the conceivable isomers. In this study, gas chromatography-tandem mass spectrometry (GC-MS-MS) was used to differentiate the regioisomers of chloroamphetamine analogs (chloroamphetamines and chloromethamphetamines) synthesized in the authors' laboratories. Free bases, trifluoroacetyl derivatives, and trimethylsilyl derivatives were subjected to GC-MS-MS using DB-1ms, DB-5ms, and DB- $17 \mathrm{~ms}$ capillary columns, respectively. The regioisomers of chloroamphetamine analogs in all forms were well separated on the DB-5ms column. The electron ionization mass spectra of the chloroamphetamine analogs gave very little structural information for differentiation among these analogs, even after trifluoroacetyl and trimethylsilyl derivatization of the analytes. Characteristic product ions of the 2-positional isomers were observed by electron ionization-MS-MS. In contrast, chemical ionization-MSMS of the free bases provided more structural information about chloride position on the aromatic ring when $[\mathrm{M}+\mathrm{H}-$
\end{abstract}

Hiroyuki Inoue

inoue@nrips.go.jp

1 National Research Institute of Police Science, 6-3-1 Kashiwanoha, Kashiwa 277-0882, Japan

2 Technopro R\&D, Company, 1-15-1 Benten, Chuo-ku, Chiba 260-0045, Japan

3 Biochemical Toxicology Laboratory, Faculty of Pharmaceutical Sciences, Kobegakuin University, 1-1-3 Minatojima, Chuo-ku, Kobe 650-8586, Japan
$\mathrm{HCl}]^{+}$was selected as a precursor ion. The results suggest that a combination of chromatographic analysis and MSMS supports differentiation for regioisomers of chloroamphetamine analogs.

Keywords Chloroamphetamine analogs $\cdot$ Regioisomeric differentiation · GC-MS-MS · Chemical ionization

\section{Introduction}

In recent years, a large number of controlled substance analogs with high structural variety have been widely distributed as noncontrolled alternatives and have been detected in forensic samples. Because of the frequent appearance of new analogs with displacement of a functional group or an alkyl chain [1-3], structural differentiation of these new drugs is necessary in forensic laboratories for legal control against drug abuse [4-14].

4-Chloromethamphetamine (4-CMA) was first reported as a selective serotoninergic depleting agent in rat brains in 1963 by Pletsher et al. [15]. Chlorinated analogs of amphetamine were subsequently synthesized and examined in the Lilly research laboratory as appetite depressants $[16$, 17]. It was eventually concluded that these analogs were not clinically useful and produced less central-nervous system stimulation than amphetamine or methamphetamine. After these reports were published, many researchers studied chloroamphetamines (CAPs) as factors of selective serotonin (5-hydroxytriptamine, 5-HT) diminution and as monoamine oxidase (MAO) inhibitors in brain nerve terminals [18-20]. It was also reported that 4-CAP and 4-CMA cause long-lasting depletion in the level of brain 5-HT and 5-hydroxyindole-3-acetic acid without depleting brain noradrenaline [21, 22]. Administration of 
4-CAP to rats induced tremors, rigidity, Straub tail, hind limb abduction, lateral head weaving, and reciprocal forepaw treading [23]. It was previously reported that the half-life of 4-CAP was longer than that of 4-fluoroamphetamine (4-FAP) in rat brains, and that the liposolubility of 4-CAP was higher than that of 4-FAP in vitro [24]. Because of a tendency to dissolve in the organic phase, the depletion of brain 5-HT with 4-CAP continued for at least a week [24].

In Taiwan, CAP first appeared in April 2009, and a total of 775 cases were identified up to 2011 [25]. 4-CAP was also detected in urine samples in March 2010 [26]. In Hungary, it was detected in December 2012 as a new psychoactive substance and reported to the European Monitoring Centre for Drugs and Drug Addiction (EMCDDA) and Europol for the first time [1]. Although there have been no reports of chloroamphetamine analogs (CAPs and CMAs) being abused and detected in forensic samples in Japan, 4-CAP has been controlled as a designated substance since August 2014.

For forensic purposes, differentiation of the chloro group ring position in amphetamine and methamphetamine is required because 4-CAP is controlled but 2-CAP and 3-CAP are not (Fig. 1). Although the results of infrared spectroscopy and nuclear magnetic resonance (NMR) spectrometry can provide structural information for identification of a specific isomer, these methods are not suitable for biological samples such as urine and blood because of their low sensitivity.

Gas chromatography-mass spectrometry (GC-MS) is generally used for the identification of drugs because it is<smiles>CC(N)Cc1ccccc1Cl</smiles>

1) 2-Chloroamphetamine (2-CAP)<smiles>CC(N)Cc1cccc(Cl)c1</smiles>

2) 3-Chloroamphetamine (3-CAP)<smiles>CC(N)Cc1ccc(Cl)cc1</smiles>

3) 4-Chloroamphetamine (4-CAP)

Fig. 1 Chemical structures regioisomers usually supported by an extensive electron ionization-mass spectrometry (EI-MS) database.

In this study, differentiation of chloroamphetamine analog regioisomers was studied using GC-MS-MS with EI and chemical ionization (CI). The effectiveness of analyte derivatization was also evaluated.

\section{Materials and methods}

\section{Chemicals}

4-Chlorophenylacetone, methylamine hydrochloride, and sodium cyanoborohydride were purchased from Tokyo Chemical Industry (Tokyo, Japan). Ortho- and $m$ chlorophenylacetone and trifluoroacetic anhydride (TFAA) were purchased from Wako Pure Chemical Industries (Osaka, Japan). N,O-Bis(trimethylsilyl)trifluoroacetamide (BSTFA) with $1 \%$ trimethylchlorosilane (TMCS) was obtained from Supelco (St Louis, MO, USA). All other chemicals were of analytical grade.

\section{Chloroamphetamine synthesis}

Chlorophenylacetone and ammonium acetate or methylamine hydrochloride (5 equiv.) were dissolved in methanol at room temperature and the solution was stirred for $1 \mathrm{~h}$. Sodium cyanoborohydride ( 0.4 equiv.) at $0{ }^{\circ} \mathrm{C}$ was added to the mixture. After the reaction was complete (Fig. 2), the $\mathrm{pH}$ of the mixture was adjusted to 12 with a $10 \%$ potassium hydroxide aqueous solution. The mixture was extracted with diethyl ether, washed with water, and dried over sodium sulfate. The extract was then evaporated under reduced pressure. The crude residue was purified by column chromatography (silica gel, chloroform:methanol $=10: 1$ ).

Chemical structures were elucidated using ${ }^{1} \mathrm{H}$ NMR spectroscopy. ${ }^{1} \mathrm{H}$ NMR spectra were measured in deuterochloroform $\left(\mathrm{CDCl}_{3}\right)$ with a JNM-ECP400 spectrometer (JEOL, Akishima, Japan) at room temperature. Tetramethylsilane was used as an internal standard. The ${ }^{1} \mathrm{H}$ NMR data for each synthesized compound are summarized below.

2-CAP; ${ }^{1} \mathrm{H}$ NMR $\left(\mathrm{CDCl}_{3}\right) \quad \delta: 7.37-7.35 \quad(1 \mathrm{H}, \mathrm{m})$, 7.22-7.14 (3H, m), 3.32-3.24 (1H, m), $2.86(1 \mathrm{H}, \mathrm{dd}$,

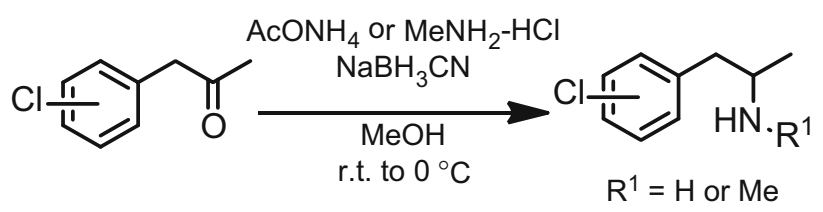

Fig. 2 Synthesis of chloroamphetamine analog regioisomers 
(a) DB-1ms

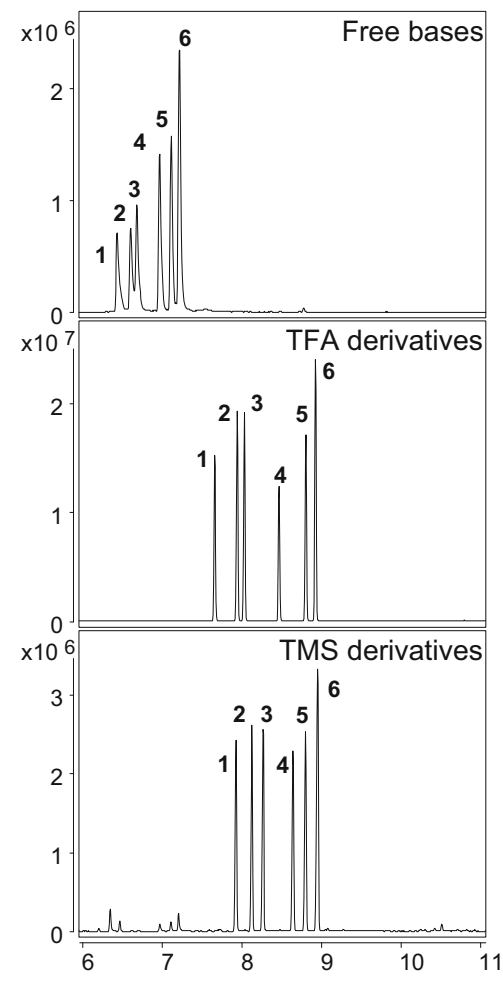

(b) DB-5ms

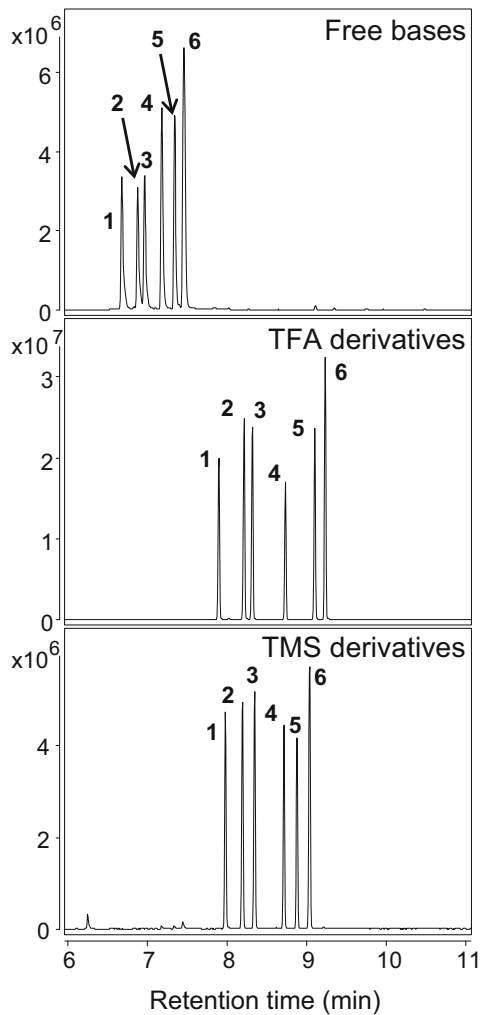

(c) DB-17ms



Fig. 3 Total ion current chromatograms for free bases, trifluoroacetyl (TFA) derivatives, and trimethylsilyl (TMS) derivatives of chloroam-

$17 \mathrm{~ms}$ (c) columns. The compound names are the same as those in phetamine analogs separated on DB-1ms (a), DB-5ms (b), and DB-

Table 1 Retention indices of chloroamphetamine analogs in different chemical forms on different separation columns

\begin{tabular}{|c|c|c|c|c|c|c|c|c|c|}
\hline \multirow[t]{3}{*}{ Analyte } & \multicolumn{9}{|c|}{ Retention index } \\
\hline & \multicolumn{3}{|l|}{ Free base } & \multicolumn{3}{|c|}{ TFA derivative } & \multicolumn{3}{|c|}{ TMS derivative } \\
\hline & DB-1ms & DB-5ms & DB-17ms & DB-1ms & DB-5ms & DB- $17 \mathrm{~ms}$ & DB-1ms & DB-5ms & DB-17ms \\
\hline 2-CAP & 1281 & 1310 & 1524 & 1426 & 1449 & 1651 & 1449 & 1455 & 1568 \\
\hline 3-CAP & 1299 & 1331 & 1546 & 1462 & 1488 & 1696 & 1473 & 1481 & 1591 \\
\hline 4-CAP & 1307 & 1341 & 1555 & 1473 & 1500 & 1707 & 1490 & 1498 & 1605 \\
\hline 2-CMA & 1340 & 1365 & 1566 & 1522 & 1549 & 1772 & 1537 & 1544 & 1658 \\
\hline 3-СMA & 1356 & 1383 & 1579 & 1566 & 1594 & 1822 & 1557 & 1563 & 1675 \\
\hline 4-CMA & 1368 & 1395 & 1590 & 1582 & 1613 & 1837 & 1576 & 1584 & 1690 \\
\hline
\end{tabular}

TFA trifluoroacetyl, TMS trimethylsilyl, CAP chloroamphetamine, CMA chloromethamphetamine

$J=13.2,6.0 \mathrm{~Hz}), 2.68(1 \mathrm{H}, \mathrm{dd}, J=13.2,8.0 \mathrm{~Hz}), 1.14$ $(3 \mathrm{H}, \mathrm{d}, J=6.4 \mathrm{~Hz})$.

3-CAP; ${ }^{1} \mathrm{H} \quad \mathrm{NMR}\left(\mathrm{CDCl}_{3}\right) \quad \delta: 7.21-7.18 \quad(3 \mathrm{H}, \mathrm{m})$, 7.08-7.06 $(1 \mathrm{H}, \mathrm{m}), 3.21-3.13(1 \mathrm{H}, \mathrm{m}), 2.68(1 \mathrm{H}, \mathrm{dd}$, $J=13.2,5.6 \mathrm{~Hz}), 2.51(1 \mathrm{H}, \mathrm{dd}, J=13.2,8.0 \mathrm{~Hz}), 1.12$ $(3 \mathrm{H}, \mathrm{d}, J=6.4 \mathrm{~Hz})$.

4-CAP; ${ }^{1} \mathrm{H} \quad \mathrm{NMR}\left(\mathrm{CDCl}_{3}\right) \quad \delta: 7.28-7.26 \quad(2 \mathrm{H}, \mathrm{m})$, 7.13-7.10 (2H, m), 3.19-3.11 (1H, m), $2.67(1 \mathrm{H}, \mathrm{dd}$,
$J=13.6,5.6 \mathrm{~Hz}), 2.52(1 \mathrm{H}, \mathrm{dd}, J=13.2,7.6 \mathrm{~Hz}), 1.05$ $(3 \mathrm{H}, \mathrm{d}, J=6.4 \mathrm{~Hz})$.

2CMA; ${ }^{1} \mathrm{H}$ NMR $\left(\mathrm{CDCl}_{3}\right) \quad \delta: 7.36-7.34(1 \mathrm{H}, \mathrm{m})$, 7.23-7.13 (3H, m), 2.97-2.87 (2H, m), 2.71-2.64 $(1 \mathrm{H}$, m), $2.44(3 \mathrm{H}, \mathrm{s}), 1.05(3 \mathrm{H}, \mathrm{d}, J=6.4 \mathrm{~Hz})$.

$3-\mathrm{CMA} ;{ }^{1} \mathrm{H}$ NMR $\left(\mathrm{CDCl}_{3}\right) \quad \delta: 7.24-7.18(3 \mathrm{H}, \mathrm{m})$, 7.10-7.06 (1H, m), 2.83-2.69 (2H, m), 2.60-2.55 $(1 \mathrm{H}$, m), $2.40(3 \mathrm{H}, \mathrm{s}), 1.05(3 \mathrm{H}, \mathrm{d}, J=5.6 \mathrm{~Hz})$. 


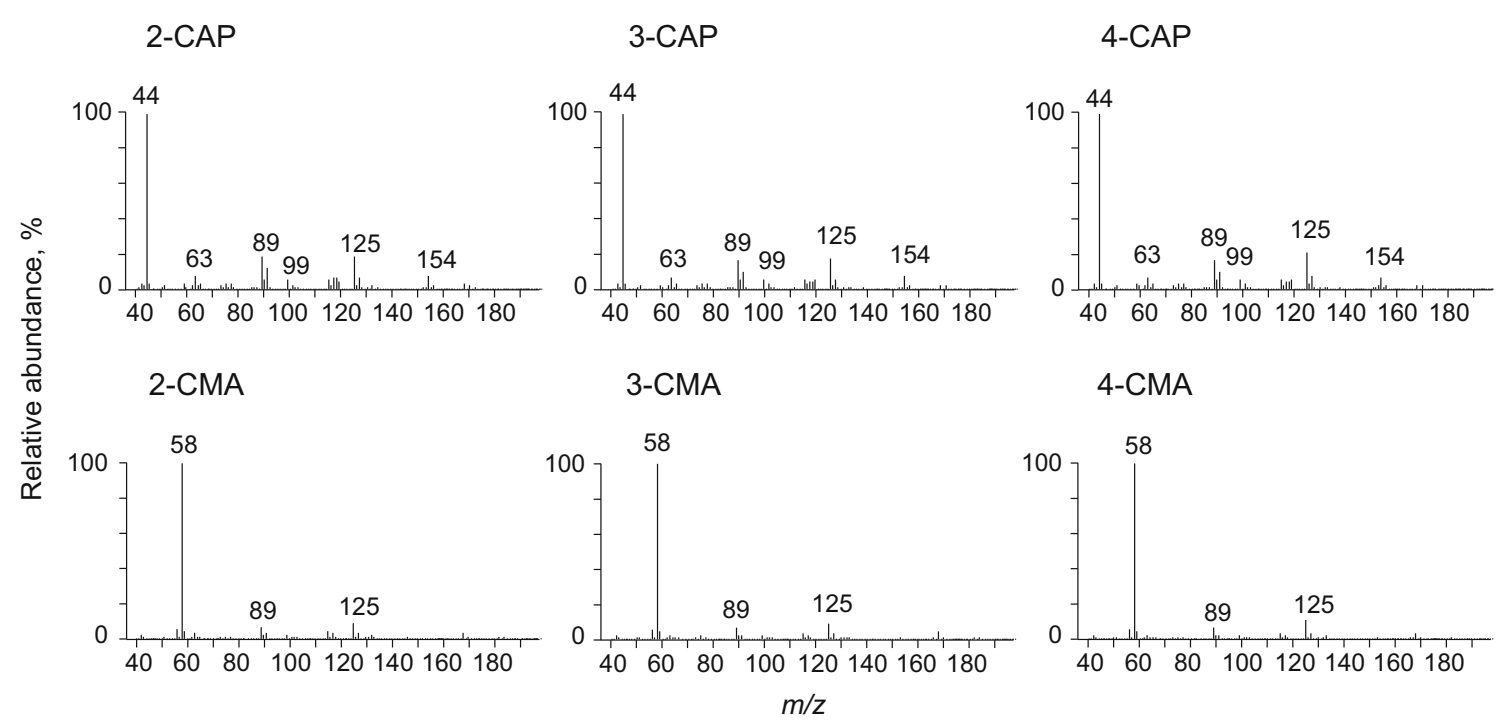

Fig. 4 Electron ionization (EI) mass spectra for free bases of chloroamphetamine analogs

(a) $m / z 154($ CE30) >

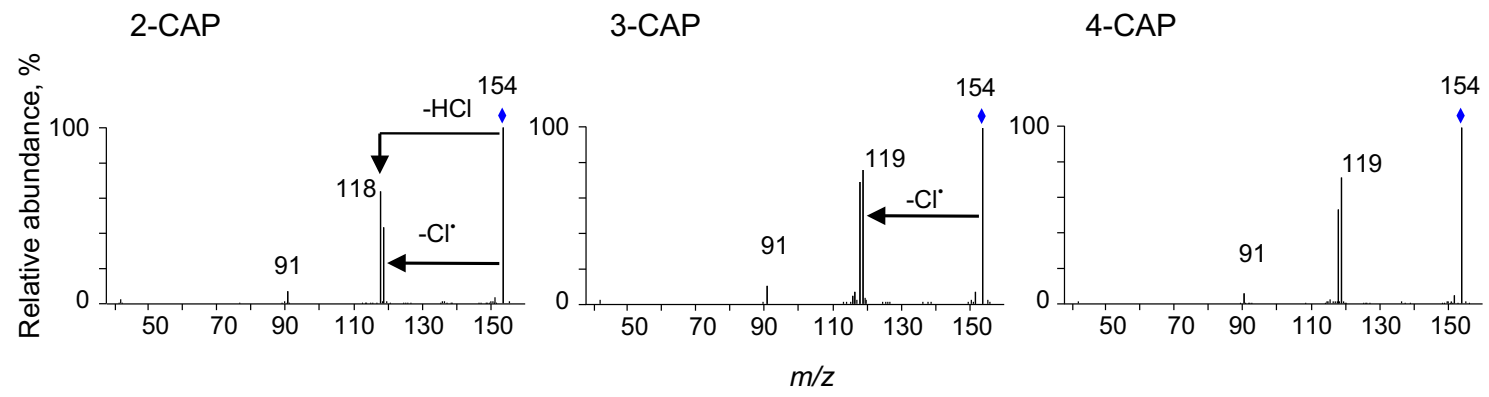

(b) $m / z 168($ CE30) >



3-CMA

4-CMA

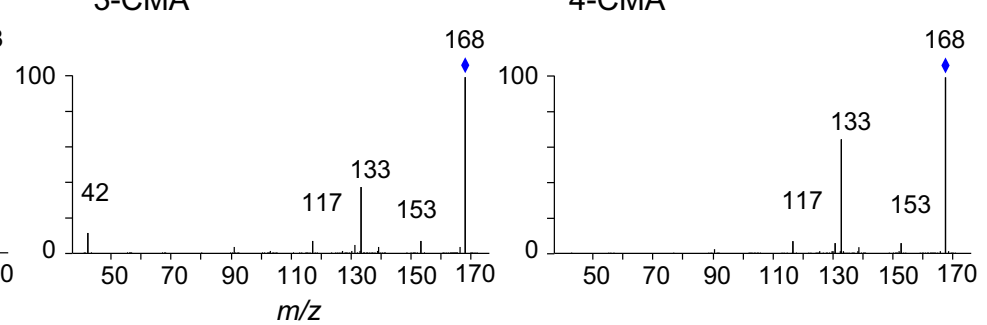

Fig. 5 EI product ion spectra for free bases of chloroamphetamines (CAPs) (precursor ions at $\mathrm{m} / \mathrm{z}$ 154; a) and chloromethamphetamines (CMAs) (precursor ions at $m / z, 168$; b)

4-CMA; ${ }^{1} \mathrm{H}$ NMR $\left(\mathrm{CDCl}_{3}\right) \quad \delta: 7.27-7.25 \quad(2 \mathrm{H}, \mathrm{m})$, 7.13-7.11 (2H, m), 2.83-2.70 (2H, m), 2.59 (1H, dd, $J=13.2, \quad 6.4 \mathrm{~Hz}), 2.39 \quad(3 \mathrm{H}, \quad \mathrm{s}), \quad 1.05 \quad(3 \mathrm{H}, \mathrm{d}$, $J=5.6 \mathrm{~Hz})$.

\section{Preparation of sample solution}

Stock standard solutions of all six compounds as corresponding hydrochloride salts were prepared in distilled water to give a concentration of $1.0 \mathrm{mg} / \mathrm{ml}$. The solutions were further diluted with distilled water to give appropriate concentrations.

\section{Extraction procedure}

One milliliter of $0.5 \mathrm{mg} / \mathrm{ml}$ aqueous solution containing hydrochloride salts of chloroamphetamine analogs was alkalinized with $20 \mu \mathrm{l}$ of $2 \mathrm{M}$ sodium hydroxide aqueous 
(a) $m / z 226($ CE60) $>$

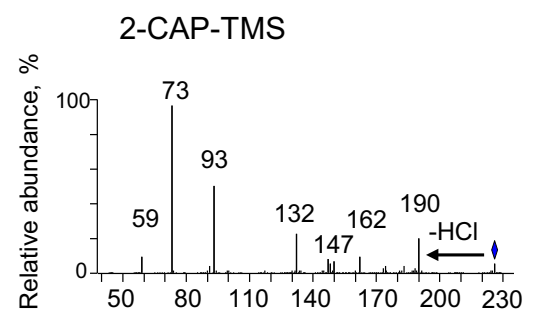

3-CAP-TMS

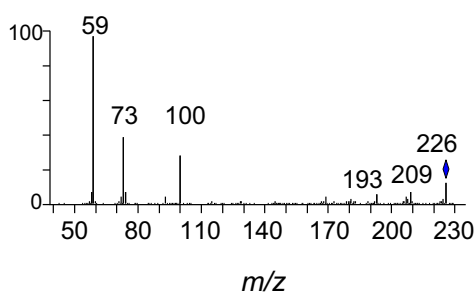

4-CAP-TMS

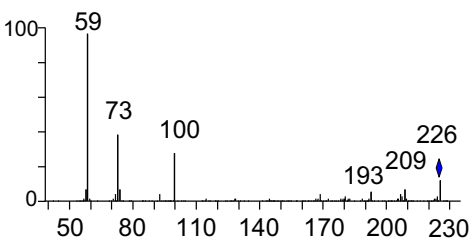

(b) $m / z 240($ CE60) $>$
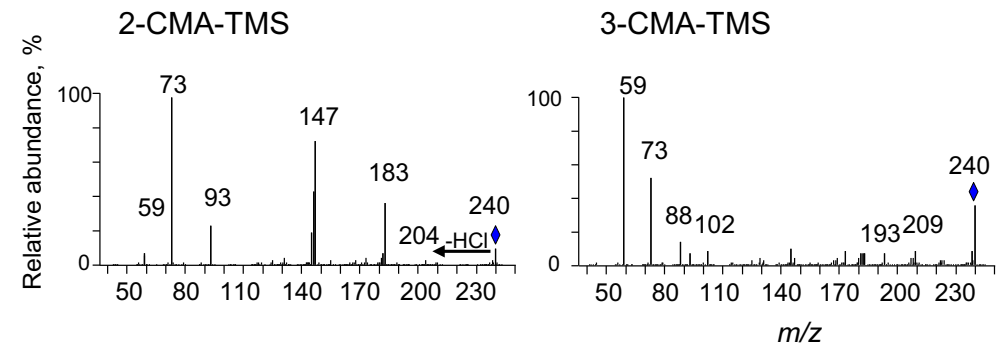

4-CMA-TMS

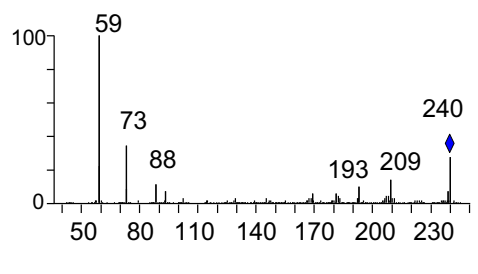

Fig. 6 EI product ion spectra for TMS derivatives of CAPs (precursor ions at $\mathrm{m} / \mathrm{z}, 226,\left[\mathrm{M}-\mathrm{CH}_{3}\right]^{+}$; a) and CMAs (precursor ions at $m / z$, 240, [M$\left.\left.\mathrm{CH}_{3}\right]^{+} ; \mathbf{b}\right)$

solution before being shaken with $1 \mathrm{ml}$ of ethyl acetate for $3 \mathrm{~min}$. After centrifugation, a portion of the extract was diluted by a factor of ten. The resulting solution was subjected to GC-MS.

\section{Trifluoroacetylation}

Up to $40 \mu$ l of ethyl acetate extract was evaporated to dryness under a gentle stream of nitrogen. The residue was dissolved in $200 \mu \mathrm{l}$ of ethyl acetate and $200 \mu \mathrm{l}$ of TFAA, and the mixture was heated for $15 \mathrm{~min}$ at $50{ }^{\circ} \mathrm{C}$. After being cooled to room temperature, the mixture was evaporated to dryness under a gentle stream of nitrogen. The residue was redissolved in $1000 \mu \mathrm{l}$ of ethyl acetate and subjected to GC-MS.

\section{Trimethylsilylation}

Ten microliters of extracted ethyl acetate solution was evaporated to dryness under a gentle stream of nitrogen. The dry residue was dissolved in $100 \mu \mathrm{l}$ of acetonitrile, and $100 \mu \mathrm{l}$ of BSTFA with $1 \%$ TMCS content was added to
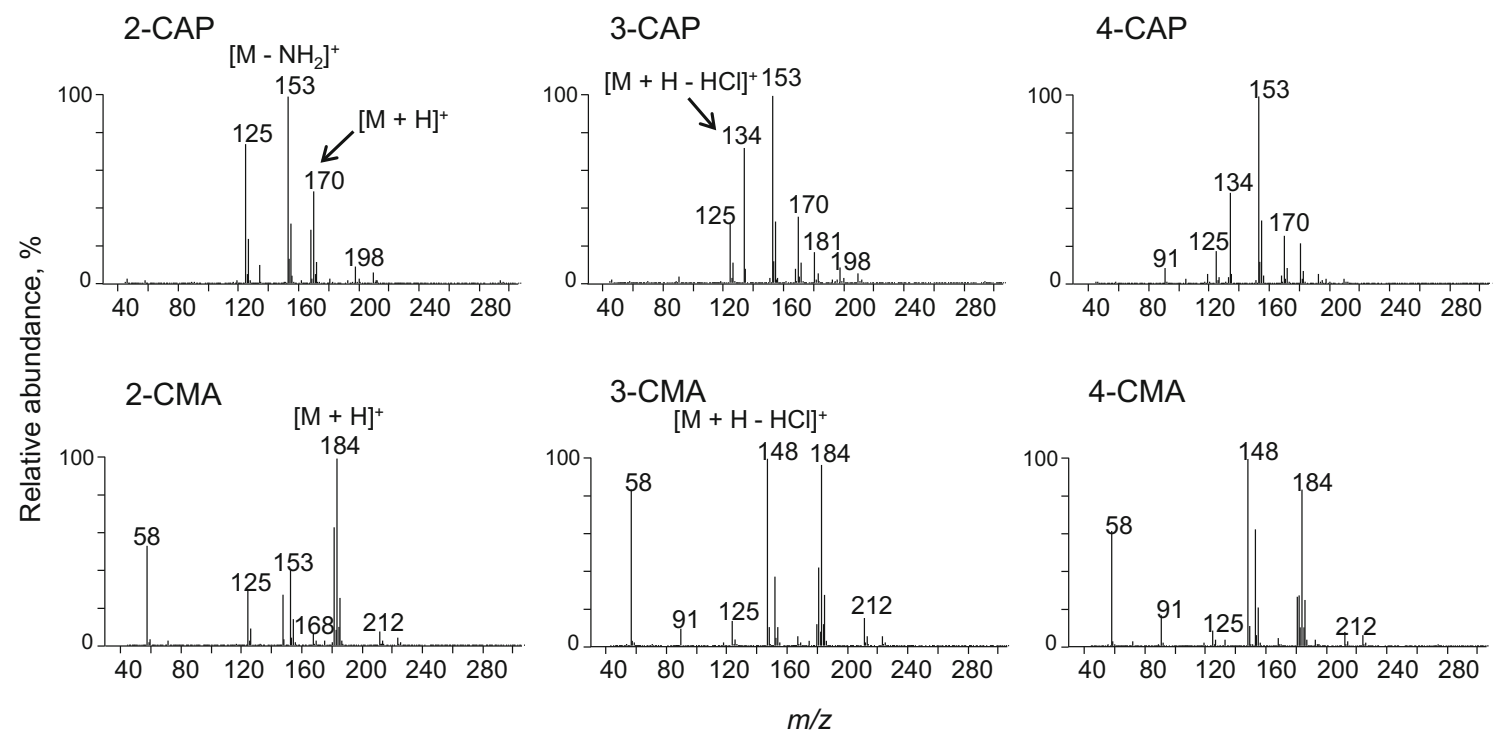

Fig. 7 Chemical ionization (CI) mass spectra for free bases of chloroamphetamine analogs 
(a) $m / z 134($ CE 20) $>$
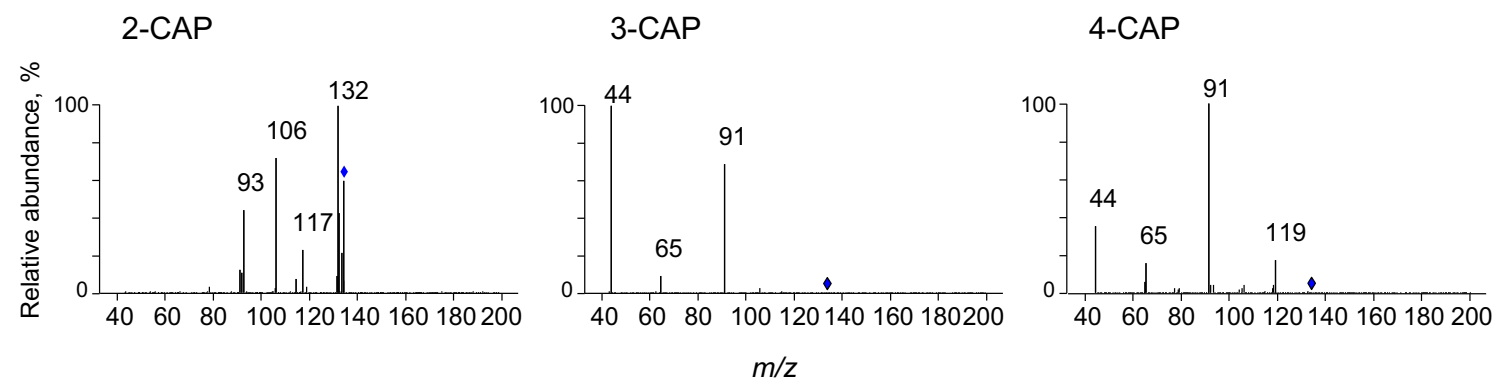

(b) $m / z 148($ CE 20) >
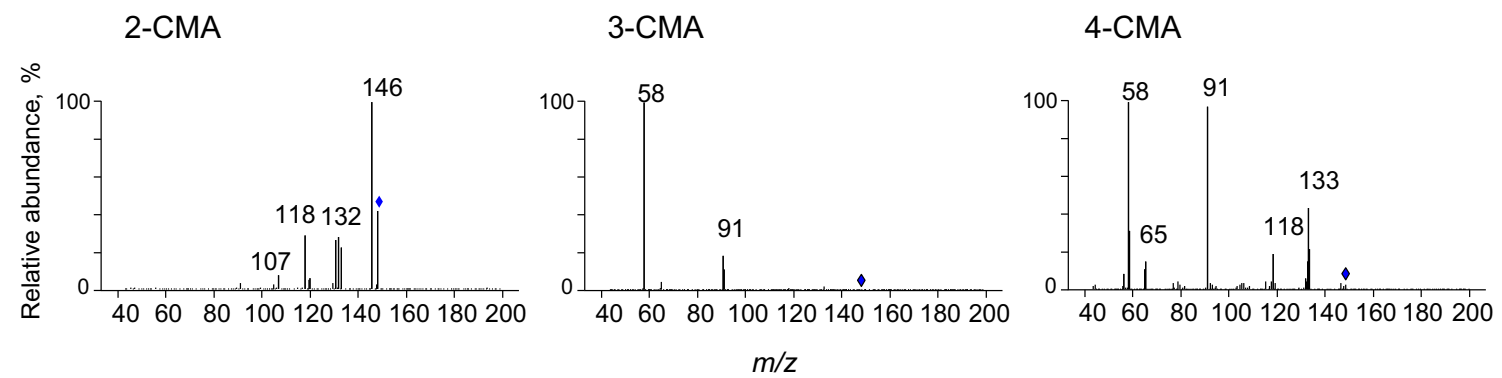

Fig. 8 CI product ion spectra for free bases of CAPs (precursor ions at $m / z, 134,[\mathrm{M}+\mathrm{H}-\mathrm{HCl}]^{+}$; a) and CMAs (precursor ions at $m / z$, 148, $[\mathrm{M}+\mathrm{H}-\mathrm{HCl}]^{+}$; b)

the solution. The mixture was heated for $30 \mathrm{~min}$ at $80{ }^{\circ} \mathrm{C}$ and subjected to GC-MS analysis after being cooled to room temperature.

\section{GC-MS conditions}

GC-MS was performed on a 7000 Triple quadrupole GCMS system (Agilent, Santa Clara, CA, USA) equipped with a DB-1ms, a DB- $5 \mathrm{~ms}$, or a DB- $17 \mathrm{~ms}$ capillary column (30 m $\times 0.25 \mathrm{~mm}$ I.D., film thickness $0.25 \mu \mathrm{m}$, Agilent $\mathrm{J} \& \mathrm{~W}$, Folsom, CA, USA). The oven temperature was maintained at $80{ }^{\circ} \mathrm{C}$ for 1 min following injection and then raised to $300{ }^{\circ} \mathrm{C}$ at a rate of $15{ }^{\circ} \mathrm{C} / \mathrm{min}$. The injection port and interface temperature were set at $250{ }^{\circ} \mathrm{C}$. Helium was used as the carrier gas at a flow rate of $1.0 \mathrm{ml} / \mathrm{min}$. One microliter of sample solution was injected in splitless mode.

For EI mode, the ionization energy was $70 \mathrm{eV}$, the ion source temperature was $230{ }^{\circ} \mathrm{C}$, and the scan mass range was $m / z$ 40-400. For CI mode, the ionization energy was $70 \mathrm{eV}$, the ion source temperature was $250^{\circ} \mathrm{C}$, and the scan mass range was $m / z$ 43-400.

\section{Results and discussion}

\section{Gas chromatography}

Figure 3 shows total ion current chromatograms of free bases, trifluoroacetyl (TFA) derivatives, and trimethylsilyl
(TMS) derivatives of chloroamphetamine analogs. Retention indices of the analogs are shown in Table 1. The regioisomers of chloroamphetamine analogs in all the forms of free bases, TFA derivatives, and TMS derivatives were eluted in the order of 2-positional isomers $>3$-positional isomers $>4$-positional isomers. Free bases of six analytes were well separated from each other on the DB-5ms column, although the peak shapes showed tailing (Fig. 3b). Their TFA and TMS derivatives were separated completely with symmetric peaks on all three separation columns.

\section{Electron ionization-mass spectrometry}

The EI mass spectra of free bases of chloroamphetamine analogs are shown in Fig. 4. The mass spectra of the isomers were very similar. Chloroamphetamine analogs showed the same major fragment ions based on benzyl bond cleavage $(\mathrm{m} / \mathrm{z}, 125)$ and $\alpha$-cleavage $(\mathrm{m} / \mathrm{z} 44$ or 58$)$. Their TFA and TMS derivatives also gave very little structural information for differentiation among these analogs (data not shown).

Tandem MS was applied under various conditions of collision energy. The characteristic fragmentation of 2-positional isomers was observed clearly from the product ion spectra of chloroamphetamine analogs with moderate collision energy ( $\mathrm{CE}=30$, Fig. 5). The fragment ions at $\mathrm{m} / \mathrm{z} 118$ and 132 based on desorption of hydrochloride came from precursor ions at $m / z, 154$ and 168. Meanwhile, 3- and 4-positional isomers produced fragment ions at 
Fig. 9 Plausible structures of the precursor ions for CItandem mass spectrometry of CAPs and CMAs and their cleavage pathways. The precursor ions were $\mathrm{m} / \mathrm{z}, 134$ for CAPs and $m / z, 148$ for CMAs, which corresponded to $[\mathrm{M}+\mathrm{H}-$ $\mathrm{HCl}]^{+}$

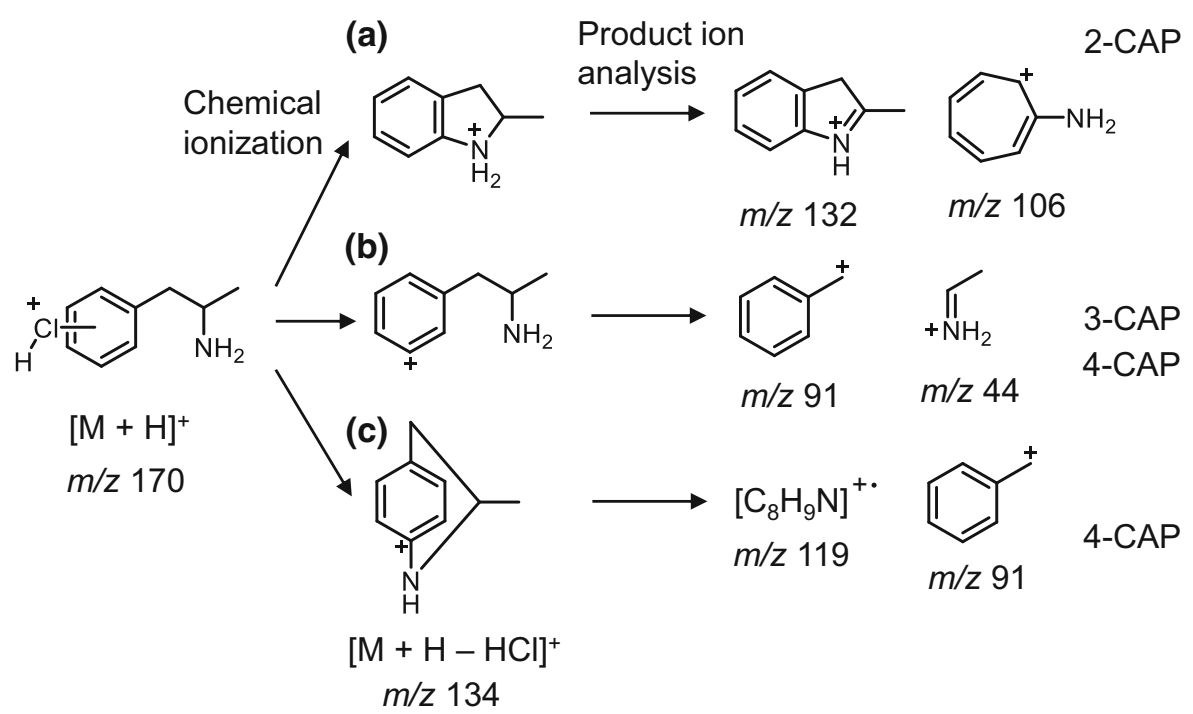

$\mathrm{m} / \mathrm{z} 119$ and 133 based on chloride radical desorption from precursor ions rather than fragment ions of hydrochloride loss under the same collision energy. The loss of hydrochloride by the 2-positional $\mathrm{Cl}$ group on an aromatic ring in tandem MS was in accordance with the content of a previous report by Katiaho et al. [27]. The ratio of the fragment ion at $m / z 42$ for 3-CMA was higher than that for 4-CMA. The difference between 3-CMA and 4-CMA was more obvious because of strong collision energy (data not shown). The results obtained here suggest that careful analysis of tandem EI-MS with changes in collision energy supports the differentiation of CMA regioisomers.

TMS derivatives of 2-positional isomers showed a significant number of fragment ions at $\mathrm{m} / \mathrm{z} 132$ and 190 for 2-CAP and $\mathrm{m} / \mathrm{z}, 147$ and 204 for 2-CMA in product ion spectra (Fig. 6). However, the spectra for TMS derivatives of 3- and 4-positional isomers were almost equivalent. It was considered that the product ions at $\mathrm{m} / \mathrm{z} 132$ and 147 were produced by desorption of $\left[\mathrm{Si}\left(\mathrm{CH}_{3}\right)_{2}-\mathrm{Cl}\right]$ from the precursor ion, $\left[\mathrm{M}-\mathrm{CH}_{3}\right]^{+}$. The ions at $m / z, 190$ and 204 came from hydrochloride desorption of the precursor ions.

\section{Chemical ionization-mass spectrometry}

The CI mass spectra for free bases of chloroamphetamine analogs are shown in Fig. 7. CI-MS of CAPs provided the same major fragment ions at $m / z 153$ and 170. In the spectra of CMAs, $[\mathrm{M}+\mathrm{H}]^{+}$at $\mathrm{m} / \mathrm{z} 184$ was also produced as the base peak. However, the mass spectra of 2-positional isomers were different from those of 3-and 4-positional isomers, the spectra of which were almost the same. Their fragment ion desorption of chloride ions $(\mathrm{m} / \mathrm{z} 134$ or 148$)$ was also higher than that of benzyl cations $(\mathrm{m} / \mathrm{z}, 125)$. The 2-positional isomers produced an equivalent or higher ratio of benzyl cations than fragment ion produced by desorption of chloride ions. TFA and TMS derivatives were examined, but only TMS derivatives of 2-CAP were differentiated by a 
(a) $m / z 226($ CE 60) $>$

\section{2-CAP-TMS}



(b) $m / z 240($ CE 60) $>$

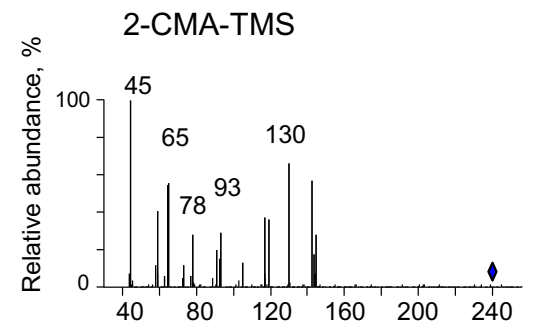

3-CAP-TMS

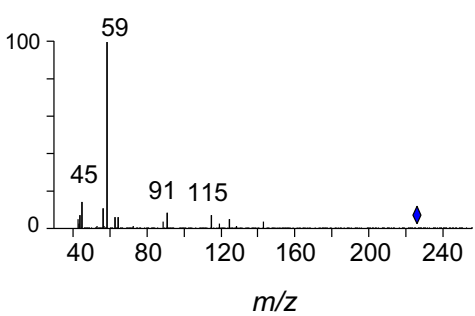

3-CMA-TMS

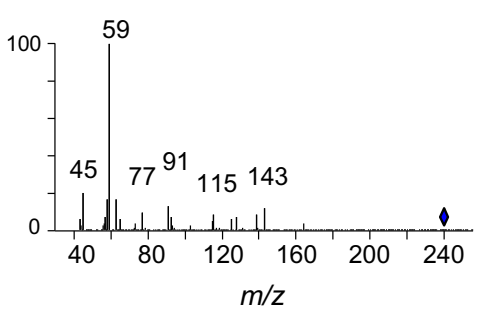

4-CAP-TMS

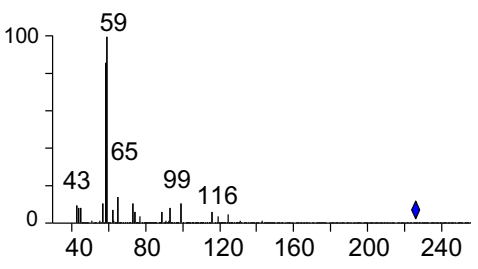

4-CMA-TMS

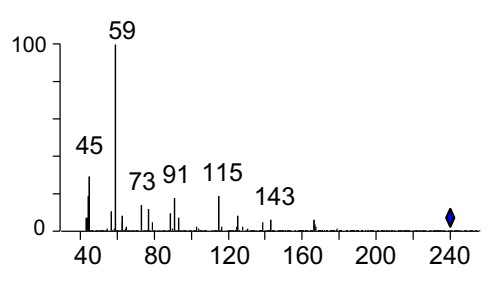

Fig. 10 CI product ion spectra for TMS derivatives of CAPs (precursor ions at $m / z, 226,\left[\mathrm{M}_{-} \mathrm{CH}_{3}\right]^{+}$; a) and CMAs (precursor ions at $m / z$ 240, $\left.\left[\mathrm{M}-\mathrm{CH}_{3}\right]^{+} ; \mathbf{b}\right)$

characteristic ion at $\mathrm{m} / \mathrm{z} 206,[\mathrm{M}+\mathrm{H}-\mathrm{HCl}]^{+}$(data not shown).

In contrast, product ion analysis of the free bases provided more structural information about chloride position on the aromatic ring when an optimal precursor ion was selected. Figure 8a shows the product ion spectra of CAP free bases obtained from the precursor ions at $\mathrm{m} / \mathrm{z} 134$, corresponding to $[\mathrm{M}+\mathrm{H}-\mathrm{HCl}]^{+}$. Three CAP isomers showed different spectrum patterns. In particular, 2-CAP was markedly different from the other isomers. Only 2-CAP gave the ions at $\mathrm{m} / \mathrm{z} 132$ (neutral loss of hydrogen molecule), 106, and 93. In addition, 2-CAP did not give the ions at $\mathrm{m} / \mathrm{z} 44$ ( $\alpha$-cleavaged iminium cation) and 91 (tropylium cation), which were observed for both 3-CAP and 4-CAP. These differences suggested that the precursor ions at $\mathrm{m} / \mathrm{z}, 134$ in the CI mass spectra were composed of different chemical structures between 2-CAP and the others. The precursor ion of 2-CAP was presumed to be 2-methylindolinium ion (Fig. 9a). This structure was supported by the ion at $\mathrm{m} / \mathrm{z} 106$ in the product ion spectrum because this ion corresponded to anchimeric-stabilized amine tropylium cation generated by rearrangement with loss of ethylene from 2-methylindolium ion. This pathway was as reported in a previous study by Westphal et al. [28] on the differentiation of fluorinated amphetamines using GC-CI-MS-MS.

The ions at $m / z, 91$ and 44 in the product ion spectra of 3-CAP and 4-CAP suggested that their precursor ions included the cations that were produced by simple elimination of $\mathrm{HCl}$ (Fig. 9b). These cations were cleaved to tropylium cation and iminium cation in product ion analysis (Fig. 9b). However, the ratio of $\mathrm{m} / \mathrm{z} 91$ against $\mathrm{m} / \mathrm{z} 44$ in 4-CAP was higher than that in 3-CAP, and their ratios were highly reproducible with a relative standard deviation (RSD) of less than $2 \%$. Moreover, the ion at $\mathrm{m} / \mathrm{z} 119$ was observed only in the product ion spectrum of 4-CAP. These findings suggested that the ions at $m / z, 134$ in the CI spectra of 4-CAP was partially derived from a para-cyclophanelike cation (Fig. 9c). The ion at $\mathrm{m} / z, 119$ in the product ion spectrum of 4-CAP was produced by loss of methyl cation from the para-cyclophane-like cation. Moreover, considering the difference in the ratio of $\mathrm{m} / \mathrm{z} 91$ and 44 between 4-CAP and 3-CAP, the tropylium cation of 4-CAP may be partially derived from the para-cyclophane-like cation.

Product ion spectra of CMAs differed between isomers with the same tendency as CAPs. Figure $8 \mathrm{~b}$ shows the product ion spectra of CMA free bases obtained from the precursor ions at $\mathrm{m} / \mathrm{z} 148$, corresponding to $[\mathrm{M}+\mathrm{H}-\mathrm{HCl}]^{+}$. 2-CMA showed the ions at $\mathrm{m} / \mathrm{z} 146,132$, and 107, which were not observed in the spectra of 3-CMA and 4-CMA. 2-CMA did not give the ions at $\mathrm{m} / \mathrm{z} 58$ ( $\alpha$-cleavaged iminium cation) and 91 (tropylium cation), which were observed for both 3-CMA and 4-CMA. The ratios of $\mathrm{m} /$ $z 91$ and 58 were different between 3-CMA and 4-CMA with high reproducibility (RSD less than $2 \%$ ). 4-CMA gave a characteristic ion of $m / z 133$, corresponding to the 
loss of methyl cation from the precursor ion. These findings suggested the ions at $\mathrm{m} / \mathrm{z}, 148$ in the CI spectra originated from 2-methylindolinium for 2-CMA (Fig. 9d), a cation which was produced by simple elimination of $\mathrm{HCl}$ for 3-CMA (Fig. 9e), and the simply eliminated cation and para-cyclophane-like cation for 4-CMA (Fig. 9e, f).

The CI product ion spectra of TMS derivatives of 2-positional isomers were clearly different from those of 3- and 4-positional isomers (Fig. 10). In contrast, the CI-MS-MS of TFA derivatives gave almost the same spectra (data not shown). These results indicate that product ion spectrometry with optimal precursor ions based on CI-MS-MS can be used to differentiate between chloroamphetamine analogs.

\section{Conclusions}

Six ring-substituted chloroamphetamine analogs were successfully separated using GC-MS with DB-1ms, DB$5 \mathrm{~ms}$, and DB-17ms columns. EI-MS provided structural information corresponding to the position of a chloride atom on the aromatic ring. All regioisomers of chloroamphetamine analogs as free bases could be differentiated using CI-MS-MS on $[\mathrm{M}+\mathrm{H}-\mathrm{HCl}]^{+}$. The results suggest that a combination of chromatographic analysis and tandem MS allow the differentiation of regioisomers of chloroamphetamine analogs.

Acknowledgments This work was supported in part by the R\&D Program for Implementation of Anti-Crime and Anti-Terrorism Technologies for a Safe and Secure Society and by Funds for Integrated Promotion of Social System Reform and Research and Development (provided by Japan's Ministry of Education, Culture, Sports, Science and Technology).

Conflict of interest There are no financial or other considerations that could lead to a conflict of interest in relation to this study.

Ethical approval This article does not contain any studies with human participants or animals.

\section{References}

1. EMCDDA-Europol (2012) Annual report on the implementation of council decision. 2005/387/JHA. doi: 10.2810/99367 (http:// www.emcdda.europa.eu/attachements.cfm/att_212366_EN_EM CDDA-Europol\%202012\%20Annual\%20Report_final.pdf)

2. INCB. annual report (2013) New psychoactive substances and other non-scheduled chemicals represent a clear and present danger. https://www.incb.org/documents/Publications/Annual Reports/AR2013/English/AR_2013_E.pdf

3. EU drug market report. strategic analysis. doi:102.2810/85143 (http://www.emcdda.europa.eu/attachements.cfm/att_194336_EN_ TD3112366ENC.pdf)

4. Pirisi MA, Nieddu M, Burrai L, Carta A, Briguglio I, Baralla E, Demontis MP, Varoni MV, Boatto G (2013) An LC-MS-MS method for quantitative analysis of six trimethoxyamphetamine designer drugs in rat plasma, and its application to a pharmacokinetic study. Forensic Toxicol 31(2):197-203

5. Nakazono Y, Tsujikawa K, Kuwayama K, Kanamori T, Iwata YT, Miyamoto K, Kasuya F, Inoue H (2013) Differentiation of regioisomeric fluoroamphetamine analogs by gas chromatography-mass spectrometry and liquid chromatography-tandem mass spectrometry. Forensic Toxicol 31:241-250

6. Zaitsu K, Miyagawa H, Sakamoto Y, Matsuta S, Tsuboi K, Nishioka H, Katagi M, Sato T, Tatsuno M, Tsuchihashi H, Suzuki $\mathrm{K}$, Ishii A (2013) Mass spectrometric differentiation of the isomers of mono-methoxyethylamphetamines and mono-methoxydimethylamphetamines by GC-EI-MS-MS. Forensic Toxicol 31:292-300

7. Nakazono Y, Tsujikawa K, Kuwayama K, Kanamori T, Iwata YT, Miyamoto K, Kasuya F, Inoue H (2014) Simultaneous determination of tryptamine analogs in designer drugs using gas chromatography-mass spectrometry and liquid chromatographytandem mass spectrometry. Forensic Toxicol 32:154-161

8. Negishi S, Nakazono Y, Tsujikawa K, Kuwayama K, Kanamori T, Iwata YT, Miyamoto K, Kasuya F, Inoue H (2014) Differentiation of regioisomeric methylamphetamines using GC/MS (in Japanese). Jpn J Forensic Sci Tech 19:111-119

9. Belal T, Awad T, DeRuiter Clark CR (2008) GC-MS studies on acylated derivatives of 3-methoxy-4-methyl- and 4-methoxy-3methyl-phenethylamines: regioisomers related to 3,4-MDMA. Forensic Sci Int 178:61-82

10. Clark CR, DeRuiter J (1996) Chromatographic and mass spectrometric methods for the differentiation of N-methyl-1-(3,4methylendioxyphenyl)-2-butanomine from regioisomeric derivatives. J Chromatogr Sci 34:230-237

11. Borth S, Hansel W, Rosner P, Junge T (2000) Synthesis of 2,3and 3,4-methylendioxyphenylalkylamines and their regioisomeric differentiation by mass spectral analysis using GC-MS-MS. Forensic Sci Int 114:139-153

12. Zaitsu K, Katagi M, Kamata HT, Miki A, Tsuchihashi H (2008) Discrimination and identification of regioisomeric $\beta$-keto analogues of 3,4-methylendioxyamphetamines by gas chromatography-mass spectrometry. Forensic Toxicol 26:45-51

13. Zaitsu K, Katagi M, Kamata H, Kamata T, Shima N, Miki A, Iwamura T, Tsuchihashi $\mathrm{H}$ (2008) Discrimination and identification of the six aromatic positional isomers of trimethoxyamphetamine (TMA) by gas chromatography-mass spectrometry (GC-MS). J Mass Spectrom 43:528-534

14. Kusano M, Zaitsu K, Nakayama H, Nakajima J, Hisatsune K, Moriyasu T, Matsuta S, Katagi M, Tsuchihashi H, Ishii A (2015) Positional isomer differentiation of synthetic cannabinoid JWH081 by GC-MS/MS. J Mass Spectrom 50:586-591

15. Pletsher A, Burkhard WP, Bruderer H, Gey KF (1963) Decrease of cerebral 5-hydroxytryptamine and 5-hydroxyindoleacetic acid by an arylalkylamine. Life Sci 2:828-833

16. Owen JE Jr (1963) Psychopharmacological studies of some 1-(chlorophenyl)-2-aminopropanes. I. Effects on appetite-controlled behavior. J Pharm Sci 52:679-683

17. Owen JE Jr (1963) Psychopharmacological studies of some 1-(chlorophenyl)-2-aminopropanes. II. Effects on avoidance and discrimination behavior. J Pharm Sci 52:684-688

18. Harvey JA, McMaster SE, Yunger LM (1975) p-Chloroamphetamine: selective neurotoxic action in brain. Science 187:841-843

19. Ögren SO, Ross SB (1977) Substituted amphetamine derivatives. II. Behavioral effects in mice related to monoaminergic neurones. Acta Pharmacol Toxicol 41:353-368

20. Fuller RW (1992) Effects of p-chloroamphetamine on brain serotonin neurons. Neurochem Res 17:449-456

21. Miller FP, Cox RH Jr, Snodgrass WR, Maickel RP (1970) Comparative effects of $p$-chlorophenylalanine, $p$ - 
chloroamphetamine and $p$-chloro- $N$-methylamphetamine on rat brain norepinephrine, serotonin and 5-hydroxyindole-3-acetic acid. Biochem Pharmacol 19:435-436

22. Sanders-Bush E, Sulser F (1970) $p$-Chloroamphetamine: in vivo investigations on the mechanism of action of the selective depletion of cerebral serotonin. J Pharm Exp Ther 175:419-426

23. Trulson ME, Jacobs BL (1976) Behavioral evidence for the rapid release of CNS serotonin by PCA and fenfluramine. Eur J Pharmacol 36:149-154

24. Fuller RW, Baker KW, Perry KW, Molloy BB (1975) Comparison of 4-chloro-, 4-bromo and 4-fluoroamphetamine in rats: drug levels in brain and effects on brain serotonin metabolism. Neuropharmacology 14:739-746
25. Lee SF, Hsu J, Tsay WI (2013) The trend of drug abuse in Taiwan during the years 1999 to 2011. J Food Drug Anal 21:390-396

26. Lin TC, Lin DL, Lua AC (2011) Detection of p-chloroamphetamine in urine samples with mass spectrometry. J Anal Toxicol 35:205-210

27. Kotiaho T, Shay BJ, Cooks RG, Eberlin MN (1993) Electrophilic aromatic $\mathrm{Cl}^{+}$addition and $\mathrm{CO}^{\bullet+}$ substitution in the gas phase. J Am Chem Soc 115:1004-1014

28. Westphal F, Rösner P, Junge Th (2010) Differentiation of regioisomeric ring-substituted fluorophenethylamines with product ion spectrometry. Forensic Sci Int 194:53-59 